\title{
Accuracy of Fetal Transcerebellar Diameter in the Prediction of Gestational Age in Singleton Pregnancy at the Second and the Third Trimesters \\ Hanna Abd-Elhammed El-Ebeisy, Hanan Abd-Elmonem Mohammed, Basma Osman Mohammed* \\ Department of Obstetrics and Gynecology, Faculty of Medicine (for Girls), Al-Azhar University, Cairo, Egypt \\ * Corresponding author: Basma Osman Mohammed, Mobile: (+20) 01277696068, E-mail: dr.m.nasr88@gmail.com
}

\begin{abstract}
Background: Gestational age (GA) is an important parameter in obstetrics for management of pregnancy and evaluating fetal development. High incidence of perinatal mortality has been noted in patients whose accurate gestational age is not known.

Objective: The aim of this study was to evaluate whether trans-cerebellar diameter (TCD) in singleton gestations can serve as a reliable predictor of gestational age (GA) in fetuses in the second and third trimester between 14-40 weeks of pregnancy in women attending the clinic for routine ultrasound examination.

Patients and methods: This prospective study was carried out at the antenatal outpatient clinic at Al-Zahraa university Hospital and El-Sahel Teaching Hospital. This study was hold one thousand of pregnant women attending the outpatient clinic for routine ultrasound examination during second and third trimester between 14-40 weeks of pregnancy in a period from 1 December 2016 to March 2019.

Patients and methods: This prospective study included a total of one thousand of pregnant women, attending at Antenatal Outpatient Clinic, Al-Zahraa University Hospital and El-Sahel Teaching Hospital. The accuracy of the transverse Cerebellar Diameter (TCD) in the prediction of gestational age (GA) in fetuses in the second and third trimester between 14-40 weeks of pregnancy was assessed. This study was conducted between December 2016 to March 2019

Results: According to accuracy of measured gestational age according to measured parameters in relation to actual gestational age (accuracy within 2 weeks), TCD had the highest accuracy (98.7\%) than other parameters in early second trimester. Accuracy of TCD in late second trimester was $91.6 \%$ and $82 \%$ in early third trimester while in late third trimester TCD had the lowest accuracy $(68.1 \%)$.

Conclusion: TCD is an accurate parameter in estimation of gestational age as its values are in close relation with that of GA by LMP. TCD is a better predictor of the gestational age when compared to other parameters especially between (14-22) weeks.
\end{abstract}

Keywords: Transcerebellar Diameter, Prediction of Gestational Age, Singleton Pregnancy at the Second and the Third Trimesters

\section{INTRODUCTION}

Gestational age estimation is one of the important decisions which guide care during pregnancy. It is the basis for the appropriate timing of deliveries and management of complications. Studies have shown that decisions based on inaccurate gestational ages result in higher fetal and maternal morbidity and mortality ${ }^{(\mathbf{1})}$.

The traditional method of estimating gestational age, which is based on the last menstrual period, could be influenced by the regularity of menstrual cycles, especially in the immediate three months pre-conception and also by prior exposure to hormonal contraception. In women from developing countries, late "antenatal booking, the absence of accurate menstruation records and menstrual cycle irregularities are additional challenges ${ }^{(2)}$.

Sonographic estimation of gestational age is the 'gold standard' in antenatal care, with the first-trimester measurement of crown-rump-length (CRL) which is considered the most reliable index (3).

The most frequently used fetal biometric parameters after the first trimester include the biparietal diameter (BPD), femur length (FL) and abdominal circumference (AC) ${ }^{(\mathbf{1})}$.

A less commonly used parameter is the Transcerebellar Diameter (TCD), which was fortuitously found to be a useful parameter for determination of GA, while it was being evaluated for the assessment of fetal cerebellar growth and its disorders. The fetal cerebellum in the embryo appears at the end of 5th week of pregnancy and can be reliably measured using ultrasonography from the 12th to14th week of gestation till delivery (4).

The anatomical location of the cerebellum in the posterior cranial fossa makes the organ less vulnerable to external pressures on the fetal cranium (5). Studies of TCD shows that the cerebellum is the least organ affected by fetal growth abnormalities and also revealed its potential for reliable estimation of gestational age (5).

Ultrasonographically, the cerebellum, in the second trimester, can be easily viewed as a central rectangular echogenic structure (vermix) connecting two oval echoluscent structures (hemispheres) after the 14th week of gestation ${ }^{(\boldsymbol{6})}$. 
The aim of this study was to evaluate whether trans-cerebellar diameter (TCD) in singleton gestations can serve as a reliable predictor of gestational age (GA) in fetuses in the second and third trimester between 14-40 weeks of pregnancy in women attending the clinic for routine ultrasound examination.

\section{PATIENTS AND METHODS}

This prospective study included a total of one thousand of pregnant women, attending at Antenatal Outpatient Clinic, Al-Zahraa University Hospital and El-Sahel Teaching Hospital. This study was conducted between December 2016 to March 2019

Subjects were seeking for routine ultrasound examination during second and third trimester between 14-40 weeks of pregnancy. They were divided in to two groups (14-27 weeks and 28 to 42 weeks).

\section{Ethical approval and written informed consent:} An approval of the study was obtained from Al-Azhar University academic and ethical committee. Written informed consent from all the subjects were obtained.

\section{The Equipment:}

All measurements were made by scanning patients using SAMSUNG MEDISON CO., LTD digital ultrasonic diagnostic imaging system with serial number 6U-85003493 and Mindray Model: DP-30) with serial number047-005992-00-2.0.

\section{Inclusion Criteria}

1. All singleton pregnancies of 14- and 40weeks gestation with known last menstrual period.

2. Clinically suspected intrauterine growth retardation (from 28 - 40-weeks).

3. Clinically suspected macrocosmic fetus (from $28-40 \mathrm{WK})$.

\section{Exclusion criteria}

1. Congenital malformations.

2. Multiple pregnancies.

The subjects who fulfilled the inclusion criteria were subjected to the following: a) Detailed history including age, parity, LMP, GA, medical history and surgical history

b) General (blood pressure, pulse, body weight and temperature) and abdominal examination (Fundal Grip, Pelvic Grip and Leopold maneuvers).

c) Routine investigations ( $\mathrm{CBC}, \mathrm{Rh}$, random blood sugar and urine analysis).

d) Trans abdominal ultrasound to assess fetal viability, BPD, FL and TCD.

\section{The primary outcome:}

The accuracy of the transverse Cerebellar Diameter (TCD) in the prediction of gestational age (GA) in fetuses in the second and third trimester between 14-40 weeks of pregnancy.

\section{Statistical Method}

Statistical analysis was performed using SPSS for Windows version 15.0. Data were presented as range, mean and standard deviation (for parametric variables), number and proportion (for categorical variables). Association between variables will be estimated using correlation coefficient (for parametric variables). Significance level was set at 0.05 .

Data were statistically described in terms of mean \pm standard deviation ( \pm SD), median and range, or frequencies (number of cases) and percentages when appropriate. Comparison between the true gestational age (GA) and each of the measured variables was done using paired $t$ test. Agreement was tested using the interclass correlation. Frequency and percent of accurate measures within 2 weeks error was calculated for each measure within each of the GA groups. $P$ values less than 0.05 was considered statistically significant. All statistical calculations were done using computer program IBM SPSS (Statistical Package for the Social Science; IBM Corp, Armonk, NY, USA) release 22 for Microsoft Windows.

\section{RESULTS}

There was no significant influence of age and parity on all parameters in estimation of gestational age in both second and third trimesters. 
Table (1): Intraclass correlation with confidence intervals

\begin{tabular}{|c|c|c|c|c|c|c|c|}
\hline \multirow{2}{*}{\multicolumn{2}{|c|}{ Parameters }} & \multirow[t]{2}{*}{$\mathbf{N}$} & \multicolumn{2}{|c|}{ Intraclass Correlation } & \multicolumn{2}{|c|}{$\begin{array}{l}\text { 95\% Confidence } \\
\text { interval for Mean }\end{array}$} & \multirow{2}{*}{$\begin{array}{c}\mathbf{P} \\
\text { value }\end{array}$} \\
\hline & & & Cronbach's Alpha & Single Measures & Upper Bound & Lower Bound & \\
\hline \multirow{4}{*}{ GA\#GA-BPD } & $14-22$ & 308 & 0.972 & 0.943 & 0.933 & 0.956 & $\mathbf{0}$ \\
\hline & $23-26$ & 179 & 0.816 & 0.689 & 0.603 & 0.759 & $\mathbf{0}$ \\
\hline & $27-34$ & 228 & 0.940 & 0.887 & 0.856 & 0.912 & $\mathbf{0}$ \\
\hline & $35-42$ & 285 & 0.864 & 0.752 & 0.690 & 0.802 & $\mathbf{0}$ \\
\hline \multirow{4}{*}{ GA\#GA-FL } & $14-22$ & 308 & 0.913 & $\mathbf{0 . 8 3 0}$ & 0.773 & 0.872 & $\mathbf{0}$ \\
\hline & $23-26$ & 179 & 0.736 & 0.581 & 0.475 & 0.670 & $\mathbf{0}$ \\
\hline & $27-34$ & 228 & 0.950 & 0.904 & 0.878 & 0.925 & $\mathbf{0}$ \\
\hline & $35-42$ & 285 & 0.865 & 0.755 & 0.697 & 0.803 & $\mathbf{0}$ \\
\hline \multirow{4}{*}{ GA\#GA-AC } & $14-22$ & 308 & 0.826 & 0.667 & 0.510 & 0.770 & $\mathbf{0}$ \\
\hline & $23-26$ & 179 & 0.537 & 0.367 & 0.234 & 0.487 & $\mathbf{0}$ \\
\hline & $27-34$ & 228 & 0.854 & 0.742 & 0.676 & 0.795 & $\mathbf{0}$ \\
\hline & $35-42$ & 285 & 0.629 & $\mathbf{0 . 4 3 7}$ & $\mathbf{0 . 3 2 3}$ & 0.536 & $\mathbf{0}$ \\
\hline \multirow{4}{*}{ GA\#GA-TCD } & $14-22$ & 308 & 0.973 & 0.944 & 0.926 & 0.957 & $\mathbf{0}$ \\
\hline & $23-26$ & 179 & 0.725 & 0.564 & 0.456 & 0.656 & $\mathbf{0}$ \\
\hline & $27-34$ & 228 & 0.887 & 0.791 & 0.734 & 0.837 & $\mathbf{0}$ \\
\hline & $35-42$ & 285 & 0.706 & $\mathbf{0 . 5 3 0}$ & 0.430 & 0.615 & $\mathbf{0}$ \\
\hline
\end{tabular}

Table (1) shows intra-class correlation coefficient with confidence interval which shows the agreement (reliability) between the parameters to evaluate gestational age with the gestational age by LMP. In early second trimester TCD showed intra-class correlation of 0.944 which was higher than other parameters. BPD was the next accurate parameter with correlation coefficient of 0.943 . Of all the parameters, FL showed the best correlation with the gestational age in the third trimester.

Table (2): Paired test

\begin{tabular}{|c|c|c|c|c|c|c|c|c|}
\hline \multirow{2}{*}{\multicolumn{2}{|c|}{ Parameters }} & \multirow{3}{*}{\begin{tabular}{|c|}
$\mathbf{N}$ \\
308
\end{tabular}} & \multirow{2}{*}{ Mean } & \multirow{2}{*}{$\begin{array}{l}\text { Standard } \\
\text { deviation }\end{array}$} & \multicolumn{2}{|c|}{ 95\% Confidence interval for Mean } & \multirow{3}{*}{$\begin{array}{c}\begin{array}{c}\mathrm{t} \\
\text { value }\end{array} \\
-\mathbf{- 1 . 7 2 7}\end{array}$} & \multirow{3}{*}{\begin{tabular}{|c|}
$P$ \\
value \\
0.085 \\
\end{tabular}} \\
\hline & & & & & Upper Bound & Lower Bound & & \\
\hline \multirow{5}{*}{ GA\#GA-BPD } & $14-22$ & & -0.08442 & 0.85763 & -0.18057 & 0.01174 & & \\
\hline & $23-26$ & 179 & 0.11732 & 1.06142 & -0.03924 & 0.27387 & 1.479 & 0.141 \\
\hline & $27-34$ & 228 & 0.03947 & 1.24640 & -0.12318 & 0.20213 & 0.478 & 0.633 \\
\hline & $35-42$ & 285 & 0.30877 & 1.33852 & 0.15271 & 0.46484 & 3.894 & 0.000 \\
\hline & Total & 1000 & 0.09200 & 1.14580 & 0.02090 & 0.16310 & 2.539 & 0.011 \\
\hline \multirow{5}{*}{ GA\#GA-FL } & $14-22$ & 308 & -0.35135 & 1.24507 & -0.51604 & -0.18667 & -4.205 & 0.000 \\
\hline & $23-26$ & \begin{tabular}{|l|}
179 \\
\end{tabular} & \begin{tabular}{|c|}
-0.13408 \\
\end{tabular} & 1.33404 & -0.33085 & 0.06269 & -1.345 & 0.180 \\
\hline & $27-34$ & 228 & 0.08772 & 1.16151 & -0.06385 & 0.23929 & 1.140 & 0.255 \\
\hline & $35-42$ & 285 & 0.26316 & 1.34172 & 0.10672 & 0.41960 & 3.311 & 0.001 \\
\hline & Total & 1000 & \begin{tabular}{|c|}
-0.00766 \\
\end{tabular} & 1.29408 & -0.09166 & 0.07635 & -0.179 & 0.858 \\
\hline \multirow{5}{*}{ GA\#GA-AC } & $14-22$ & 308 & \begin{tabular}{|c|}
-0.59119 \\
\end{tabular} & 1.35578 & -0.80356 & -0.37883 & -4.205 & 0.000 \\
\hline & $23-26$ & 179 & 0.15084 & 1.97588 & -0.14060 & 0.44227 & 1.021 & 0.308 \\
\hline & $27-34$ & 228 & 0.31140 & 1.96768 & 0.05463 & 0.56818 & 2.390 & 0.018 \\
\hline & $35-42$ & 285 & 0.75088 & 2.43023 & 0.46752 & 1.03423 & 5.216 & 0.000 \\
\hline & Total & 1000 & 0.25617 & 2.09429 & 0.11526 & 0.39708 & 3.568 & 0.000 \\
\hline \multirow{5}{*}{ GA\#GA-TCD } & $14-22$ & 308 & \begin{tabular}{|l|}
0.021727 \\
\end{tabular} & 0.84285 & $-\mathbf{- 0 . 3 2 1 7 7}$ & -0.13277 & -1.205 & 0.000 \\
\hline & $23-26$ & 179 & 0.21788 & 1.39119 & 0.01268 & 0.42307 & 2.095 & 0.038 \\
\hline & $27-34$ & 228 & 0.37281 & $\mathbf{1 . 7 9 9 7 9}$ & 0.13794 & 0.60768 & 3.128 & 0.002 \\
\hline & $35-42$ & 285 & 0.60702 & 2.29358 & 0.33960 & 0.87444 & 4.468 & 0.000 \\
\hline & Total & 1000 & 0.22700 & 1.70422 & 0.12124 & 0.33276 & 4.212 & 0.000 \\
\hline
\end{tabular}

Table (2) shows the paired sample test of in which we will depend on mean difference for better evaluation than $\mathrm{P}$-value which is clinically significance in all parameters. Despite statistical significance, the difference is clinically not important because of large sample size. In the early second trimester, TCD had lowest mean difference 0.021727 and $p$ value (0.000). In the late second trimester, the mean difference according to BPD is 0.11732 which is less than TCD 0.21788 . FL has the least mean difference in third trimester. 
Table (3): Accuracy within two weeks

\begin{tabular}{|c|c|c|c|c|c|c|c|}
\hline Parameters & Weeks & $\begin{array}{c}\text { Frequency (N) } \\
\text { Percent }(100 \%)\end{array}$ & Accurate & Inaccurate & Total \% (measured) & Missing (N) & Total $(\%)$ \\
\hline \multirow{8}{*}{ GA\#GA-BPD } & \multirow{2}{*}{$14-22$} & $(\mathrm{~N})$ & 304 & 4 & 308 & & \\
\hline & & $(100 \%)$ & 98.5 & 1.3 & 100 & & \\
\hline & \multirow{2}{*}{$23-26$} & $(\mathrm{~N})$ & 174 & 5 & 179 & & \\
\hline & & $(100 \%)$ & 97.2 & 2.8 & 100 & & \\
\hline & \multirow{2}{*}{$27-34$} & $(\mathrm{~N})$ & 217 & 11 & 228 & & \\
\hline & & $(100 \%)$ & 95.2 & 4.8 & 100 & & \\
\hline & \multirow{2}{*}{$35-42$} & $(\mathrm{~N})$ & 263 & 22 & 285 & & \\
\hline & & $(100 \%)$ & 92.3 & 7.7 & 100 & & \\
\hline \multirow{8}{*}{ GA\#GA-FL } & \multirow{2}{*}{$14-22$} & $(\mathrm{~N})$ & 214 & 8 & 222 & 86 & 306 \\
\hline & & $(100 \%)$ & 69.5 & 2.6 & 72.1 & 27.9 & 100 \\
\hline & \multirow{2}{*}{$23-26$} & $(\mathrm{~N})$ & 168 & 11 & 179 & & \\
\hline & & $(100 \%)$ & 93.9 & 6.1 & 100 & & \\
\hline & \multirow{2}{*}{ 27- 34} & $(\mathrm{~N})$ & 216 & 12 & 228 & & \\
\hline & & $(100 \%)$ & 94.7 & 5.3 & 100 & & \\
\hline & \multirow{2}{*}{$35-42$} & $(\mathrm{~N})$ & 261 & 24 & 285 & & \\
\hline & & $(100 \%)$ & 91.6 & 8.4 & 100 & & \\
\hline \multirow{8}{*}{ GA\#GA-AC } & \multirow{2}{*}{$14-22$} & $(\mathrm{~N})$ & 146 & 13 & 159 & 149 & 308 \\
\hline & & $(100 \%)$ & 47.4 & 4.2 & 51.6 & 48.4 & 100 \\
\hline & \multirow{2}{*}{$23-26$} & $(\mathrm{~N})$ & 145 & 34 & 179 & & \\
\hline & & $(100 \%)$ & 81 & 19 & 100 & & \\
\hline & \multirow{2}{*}{ 27- 34} & $(\mathrm{~N})$ & 181 & 47 & 228 & & \\
\hline & & $(100 \%)$ & 79.4 & 20.6 & 100 & & \\
\hline & \multirow{2}{*}{$35-42$} & $(\mathrm{~N})$ & 237 & 48 & 285 & & \\
\hline & & $(100 \%)$ & 83.2 & 16.8 & 100 & & \\
\hline \multirow{8}{*}{ GA\#GA-TCD } & \multirow{2}{*}{$14-22$} & $(\mathrm{~N})$ & 304 & 4 & 308 & & \\
\hline & & $(100 \%)$ & 98.7 & 1.3 & 100 & & \\
\hline & \multirow{2}{*}{$23-26$} & $(\mathrm{~N})$ & 164 & 15 & 179 & & \\
\hline & & $(100 \%)$ & 91.6 & 8.4 & 100 & & \\
\hline & \multirow{2}{*}{ 27- 34} & $(\mathrm{~N})$ & 187 & 41 & 228 & & \\
\hline & & $(100 \%)$ & 82 & 18 & 100 & & \\
\hline & \multirow{2}{*}{$35-42$} & $(\mathrm{~N})$ & 194 & 91 & 285 & & \\
\hline & & $(100 \%)$ & 68.1 & 31.9 & 100 & & \\
\hline
\end{tabular}

According to accuracy of measured gestational age according to measured parameters in relation to actual gestational age (accuracy within 2 weeks), TCD have the highest accuracy (98.7\%) than other parameters in early second trimester. Accuracy of TCD in late second trimester is $91.6 \%$ and $82 \%$ in early third trimester while in late third trimester TCD has the lowest accuracy $(68.1 \%)$. 
Table (4): Mean and standard deviation of all parameters with confidence interval

\begin{tabular}{|c|c|c|c|c|c|c|c|c|}
\hline \multirow{2}{*}{\multicolumn{2}{|c|}{ Parameters }} & \multirow{2}{*}{$\mathbf{N}$} & \multirow{2}{*}{ Mean } & \multirow{2}{*}{$\begin{array}{l}\text { Standard } \\
\text { Deviation }\end{array}$} & \multicolumn{2}{|c|}{ 95\% CI for Mean } & \multirow{2}{*}{ Min. } & \multirow{2}{*}{ Max. } \\
\hline & & & & & Upper Bound & Lower Bound & & \\
\hline \multirow{5}{*}{ GA\#GA-BPD } & $14-22$ & 308 & 17.7013 & 2.58063 & 17.9906 & 17.4120 & 14.00 & 22.00 \\
\hline & $23-26$ & 179 & 24.8883 & 1.15575 & 25.0587 & 24.7178 & 23.00 & 26.00 \\
\hline & $27-34$ & 228 & 30.4211 & 2.44731 & 30.7404 & 30.1017 & 27.00 & 34.00 \\
\hline & $35-42$ & 285 & 38.2105 & 1.93683 & 38.4364 & 37.9847 & 35.00 & 42.00 \\
\hline & Total & 1000 & 27.7330 & 8.37591 & 28.2528 & 27.2132 & 14.00 & 42.00 \\
\hline \multirow{5}{*}{ GA\#GA-FL } & $14-22$ & 308 & 17.79 & 2.644 & 18.08 & 17.49 & 14 & 25 \\
\hline & $23-26$ & 179 & 24.77 & 1.517 & 24.99 & 24.55 & 21 & 28 \\
\hline & $27-34$ & 228 & 30.38 & 2.776 & 30.74 & 30.02 & 24 & 38 \\
\hline & $35-42$ & 285 & 37.90 & 1.933 & 38.13 & 37.68 & 32 & 42 \\
\hline & Total & 1000 & 27.64 & 8.279 & 28.15 & 27.13 & 14 & 42 \\
\hline \multirow{5}{*}{ GA\#GA-AC } & $14-22$ & 308 & 19.24 & 2.396 & 19.56 & 18.92 & 15 & 29 \\
\hline & $23-26$ & 179 & 25.02 & 1.709 & 25.27 & 24.77 & 20 & 29 \\
\hline & $27-34$ & 228 & 30.33 & 2.852 & 30.71 & 29.96 & 24 & 38 \\
\hline & $35-42$ & 285 & 37.95 & 1.948 & 38.17 & 37.72 & 32 & 42 \\
\hline & Total & 1000 & 28.97 & 7.541 & 29.46 & 28.48 & 15 & 42 \\
\hline \multirow{5}{*}{ GA\#GA-TCD } & $14-22$ & 308 & 16.37 & 1.901 & 20.67 & 20.07 & 16 & 25 \\
\hline & $23-26$ & 179 & 24.74 & 2.199 & 25.06 & 24.41 & 20 & 30 \\
\hline & $27-34$ & 228 & 30.11 & 3.037 & 30.51 & 29.71 & 23 & 37 \\
\hline & $35-42$ & 285 & 37.46 & 2.677 & 37.77 & 37.15 & 14 & 42 \\
\hline & Total & 1000 & 29.62 & 6.945 & 30.09 & 29.15 & 14 & 42 \\
\hline
\end{tabular}

When the mean GA based on BPD, HC, AC, FL and TCD were compared with that of LMP, all parameters in second trimester were showing GA which was near to that of LMP. TCD had mean GA of 16.37 in early second trimester and 24.74 in late second trimester which is very near to GA by LMP. In early third trimester TCD had mean GA of 30.11 and 37.46 in late second trimester which was very close to GA by LMP. When we compared the overall mean GA, TCD showed mean GA which was closest to that of LMP between (14-22 weeks).

\section{DISCUSSION}

The age of the included subjects (mean \pm SD) was $27.3( \pm 5.5)$ years. In our study, when mean GA based on all parameters was compared with that of LMP, TCD had mean GA of (16.37 w) and (24.74 w) in early and late second trimester. In early and late third trimester also, TCD showed mean GA (30.11 w) and $(37.6 \mathrm{w})$.

When we obtained intra-class correlation coefficient with confidence interval which shows the agreement (reliability) between the parameters to evaluate gestational age with the gestational age by LMP. In early second trimester TCD showed intra-class correlation of 0.944 which was higher than other parameters. BPD was the next accurate parameter with correlation coefficient of 0.943 . Of all the parameters, FL showed the best correlation with the gestational age in the third trimester.

By comparing the gestational age by LMP and the gestational age by other parameters, we obtained a paired $t$ test $(t)$ in which we will depend on mean difference for better evaluation than P-value which is clinically significance in all parameters. Despite statistical significance, the difference is clinically not important because of large sample size. In the second trimester, TCD had lowest mean difference 0.84285 followed BPD. In the late third trimester, the mean difference according to FL is 0.26316.

According to accuracy of measured gestational age according to measured parameters in relation to actual gestational age (accuracy within 2 weeks), TCD have the highest accuracy $(98.7 \%)$ than other parameters in early second trimester. Accuracy of TCD in late second trimester is $91.6 \%$ and $82 \%$ in early third trimester while in late third trimester TCD has the lowest accuracy $(68.1 \%)$.

In a study done by Chavez et al. ${ }^{(8)}$, the concordance between the actual and predicted gestational age by TCD was high $(\mathrm{r}=0.92 ; \mathrm{p}<.001)$. The agreement was superior in the second trimester $(\mathrm{r}=0.93)$ compared to third trimester $(r=0.81 ; \mathrm{p}<0.001)$.

Chavez et al. ${ }^{(9)}$, studied TCD in twin pregnancies and concluded that the agreement between the actual gestational age and the predicted gestational age by TCD was comparable to that of singleton pregnancy (Pearson's correlation coefficient $\mathrm{r}=0.997$, $\mathrm{p}<0.001)$.

In another study by Chavez et al. (10), the concordance between the actual gestational age and the predicted gestational age by TCD was high for both IUGR and large fetus ( $\mathrm{r}=0.98$ and 0.95 respectively).

Joshi et al. ${ }^{(11)}$ measured TCD in 594 singleton pregnancies. They found that the gestational age and 
$\operatorname{TCD}\left(50^{\text {th }}\right.$ percentile in $\left.\mathrm{mm}\right)$ coincided well till $20^{\text {th }}$ week of gestation. They observed no significant clinical difference between the nomogram created by them and the previously published nomogram in gestational age between $21^{\text {st }}$ and $28^{\text {th }}$ weeks. They observed significant differences between their nomogram and the previously published nomograms in third trimester.

Gupta et al. ${ }^{(12)}$, studied TCD in singleton pregnancies and observed that the gestational age of pregnant women not sure of their LMP can be reliably estimated by measuring the TCD which showed good correlation $(\mathrm{r}=+0.946, \mathrm{r} 2=89.6 \%$ and $\mathrm{p}<0.001)$. The increase in TCD throughout gestation helped in assessing the development of the cerebellum.

Study was done by Naseem et al. ${ }^{(13)}$ at 228 patients with gestational age of 36 weeks measuring TCD and BPD by ultrasonography. They compared GA by TCD and BPD with LMP. In this study, they observed that in 228 patients, when compared with GA by LMP, TCD had given accurate gestational age in 209 patients and BPD had given accurate gestational age in 176 patients.

Rotmensch et al. ${ }^{(14)}$, measured the cerebellar diameter in cases of down syndrome and found that cerebellar diameters in Down syndrome fetuses were lesser than normal controls at all gestational age, $(\mathrm{p}<0.005)$ by an average of $0.67-0.87 \mathrm{~mm}$. A ratio of 0.92 for observed/expected cerebellar diameter gave a sensitivity of $21 \%$, specificity of $95 \%$ and PPV $1.66 \%$ and $0.50 \%$ in a population with risk of having Down syndrome of 1 in 250 and 1 in 750 respectively. However, this difference in cerebellar size was too small to be used clinically.

Reece et al. (15) investigated the posterior cranial fossa of the fetus and confirmed the capability of the ultrasound to demonstrate the anatomy of the fetal posterior cranial fossa. They suggested that the use of fetal trans-cerebellar diameter in utero between 17 and 40 weeks of gestation is a useful indicator of accurate gestational age. Reddy et al. ${ }^{(16)}$ evaluated accuracy of predicting GA using Fetal Transcerebellar Diameter (TCD) and to compare between TCD and other existing parameters in evaluating GA in 15 to 40 weeks of gestation. They showed that TCD is an accurate parameter in estimation of gestational age in second and third trimesters as its values are in close relation with that of GA by LMP. It is also better predictor of the gestational age when compared to other parameters especially in third trimester.

Abdelmonaem et al. ${ }^{(1)}$ conclude that TCD is the most accurate method for assessment of gestational age in third trimester followed by FL, and the least accurate is the BPD. Also, by combining accuracy of TCD $(89 \%)$ and that of FL $(81 \%)$ we can be near certain of gestational age in most of our patients even if they are unsure of their dates.

\section{CONCLUSION}

It could be concluded that TCD is an accurate parameter in estimation of gestational age as its values are in close relation with that of GA by LMP. TCD is a better predictor of the gestational age when compared to other parameters especially between (14-22) weeks. The correlation between the LMP derived gestational age and the gestational age by TCD seems to decrease from second to third trimester. TCD is not affected by age of the mother and parity.

\section{REFERENCES}

1. Abdelmonaem MZ, Mohamed AS, Eldarder AK (2019): Comparison between Transcerebellar Diameter, Biparietal Diameter and Femur length for Gestational Age Measurement Accuracy in Third Trimester of Pregnancy. The Egyptian of Journal Hospital Medicine, 74: 17-22.

2. Goel P, Singla M, Ghai R, Jain S, Budhiraja V, Ramesh CS (2017): Transverse cerebellar diameter - a marker for estimation of gestational age. J Anat Soc India, 59: 158-61.

3. Oladokun A, Oladokun RE, Morhason-Bello I, Bello AF, Adedokun B (2017): Proximate predictors of early antenatal registration among Nigerian pregnant women. Ann Afr Med., 9: 222-225.

4. Kimberly B, Fredericton NB, Ken L, Vancouver BC (2014): Determination of Gestational Age by Ultrasound. J Obstet Gynaecol Can., 36(2): 171-181.

5. Nasir K, Rizwana C, Tehmina $N$ (2013): Role of Transverse Cerebellar Diameter in Diagnosis of Asymmetrical Fetal Growth Restriction. Journal of Rawalpindi Medical College (JRMC), 17(2): 231-233.

6. Satish L, Prasad BS, Likhitha S (2014): Cerebellar Measurements with Ultrasonography in the Evaluation of Fetal Age. J Dent Med Sci., 13(9): 49-56.

7. Chang CH, Chang FM, Yu CH, Ko HC, Chen HY (2017): Assessment of fetal cerebellar volume using three-dimensional ultrasound. Ultrasound Med Biol., 26: 981-988.

8. Chavez MR, Ananth CV, Smulian JC, Yeo L, Oyelese Y, Vintzileos AM (2004): Fetal transcerebellar diameter measurement with particular emphasis in the third trimester:a reliable predictor of gestational age. Am J Obstet Gynecol., 191:979-84.

9. Chavez MR, Ananth CV, Kaminsky LM, Smulian JC, Yeo L, Vintzileos AM (2006): Fetal transcerebellar diameter measurement for prediction of gestational age in twins. American Journal of Obstetrics and Gynecology, 195(6): 116771.

10. Chavez MR, Ananth CV, Smulian JC, Vintzileos AM (2007): Fetal transcerebellar diameter measurement for prediction of gestational age at the extremes of fetal growth. Journal of Ultrasound in Medicine, 26:1167-71.

11. Joshi BR (2017): Fetal transcerebellar diameter nomogram in Nepalese population. Journal of Institute of Medicine, 32(1):1923.

12. Gupta AD, Banerjee A, Rammurthy N, Revati P, Jose J (2012): Gestational age estimation using transcerebellar diameter with grading of fetal cerebellar growth. NJCA., 1(3):115-20.

13. Naseem F, Ali S, Basit U, Fatima N (2014): Assessment of gestational age,comparison between transcerebellar diameter versus femur length on ultrasound in third trimester of pregnancy. Professional Med J., 21(2):412-17.

14. Rotmensch S, Goldstein J, Liberati M, Shalev J, Ben-Rafael Z, Copel JA (2017): Fetal transcerbellar diameter in down's syndrome. Obstet Gynecol., 89(4):534-37.

15. Reece EA, Goldstein J, Pile Hoobins JC (1987): Fetal cerebellar growth unaffected by intrauterine growth retardation: a new parameter for prenatal diagnosis. AMJ Obstet Gynecol., 157: 632-638.

16. Reddy RH, Prashanth K and Ajit M (2017): Significance of fetal transcerebellar diameter in fetal biometry: A pilot study. Journal of Clinical and Diagnostic Research, 11(6): 01-04. 

ejhm.journals.ekb.eg 